\title{
Laser Shibori: A Digital Moulding Technique Supporting Circular Textile Design in Three Dimensions
}

\author{
Laura Morgan $^{1 *}$, Faith Kane ${ }^{1}$, John Tyrer ${ }^{2}$, Jinsong Shen ${ }^{3}$ \\ ${ }^{1}$ Textile Design Research Group, School of the Arts English and Drama, Loughborough University, \\ Loughborough. UK. \\ ${ }^{2}$ Wolfson School of Mechanical and Manufacturing Engineering, Loughborough University, \\ Loughborough. UK. \\ ${ }^{3}$ Textile Engineering and Materials Research Group, School of Design, De Montfort University, \\ Leicester, UK.
}

*Corresponding Author: Laura Morgan. Email: L.E.Morgan@lboro.ac.uk

Word Count: 5020

\begin{abstract}
:
This paper considers the potential for digital laser technology to facilitate sustainable innovation in the field of textile design and manufacture, enabling transition towards a circular economy. Using recent design research as a case study, it discusses a newly developed Laser Shibori technique and its significance in relation to circularity. Laser Shibori describes a digital moulding technique for threedimensional surface design and sustainable textile finishing that can be used to design accurate surface architectures for synthetic textiles. Using the photothermal energy of a $\mathrm{CO}_{2}$ laser, the method combines two heat dependent processes: heat setting and textile coloration, resulting in an effect akin to shibori. Unlike the traditional craft practice, Laser Shibori offers precise digital control, repeatability and a unique aesthetic.
\end{abstract}

The study demonstrated the benefit of interdisciplinary research, synthesising design and science to support sustainable material innovation. The synthesis of material science and creative design practice proved essential in developing the laser technique and created a platform for material innovation beyond creativity as discussed through potential functional application ideas and sustainability benefits. The methods described in this paper provide a system to control three-dimensional effects through controlled tension and targeted laser irradiation. The use of laser technology to create threedimensional textile forms presents processing advantages over traditional methods: the laser does not require physical moulds or complicated set up and offers ease of pattern change through digital generation of designs. The laser process negates requirement for additional materials, offering reversible surface design effects to facilitate ease of recovery at end of primary use, thus complimenting a circular textile lifecycle in three dimensions: through efficiency, agility and recovery.

Keywords: Circular Economy, Material Innovation, Digital Textiles, Textile Design, Laser, Sustainable Fashion.

Funding: This research was funded by the Arts and Humanities Research Council under grant reference: AH/J002666/1. 


\section{Introduction}

This paper presents a case study from an interdisciplinary, collaborative research study (Morgan, 2016), which aimed to develop new laser processing techniques for coloration, patterning and threedimensional surface design of textiles. The research examined laser technology as a tool to support sustainable innovation through science and design. The use of laser technology as a multipurpose tool for textile design and garment finishing offers environmental and economic benefits. Lasers offer digital control with potential to support sustainability through efficiency and direct-to-garment processing opportunities.

The case study details one of the developed processes: Laser Shibori, a digital moulding technique for three-dimensional surface design and sustainable textile finishing. The aim of the paper is to discuss the control and application opportunities of the Laser Shibori technique, summarising the advantages for sustainability, design, manufacture and recovery. Laser Shibori builds on the idea of mono material processing for synthetic fabrics, adding value and form to textiles through use of a new processing technique. The digital technique combines laser moulding and laser dyeing procedures transforming materials, so that design features emerge from the cloth, without affecting their ability to be recycled or reused in a later phase of their life cycle. Furthermore, the moulding features are reversible, offering a continued loop of wear and re-design to support longevity through design flexibility, customisation and re-manufacture.

\subsection{Sustainable textiles and the circular economy}

The vision for a circular economy within the textile industry can be understood as one in which textile goods, materials or fibres retain value during all phases of their life cycle, allowing them to re-enter the economy after use (The Ellen MacArthur Foundation 2017), rather than adding to the growing global problem of textile waste and its associated pollutive by-products. A circular approach to textiles should not only focus on the recovery of textile goods, but also focus on improving the sustainability of material systems including their manufacture and use.

Material choices have a significant impact at a textile's end of life. As such, the importance for designers and developers to design for recycling or disassembly has been noted (Forum for the Future 2007). Further to this, Cradle-to-Cradle (McDonough \& Braungart 2002) describes a principle by which waste is considered from the inception of new products with the aim that they can be reprocessed as raw materials at the end of their usable lifespan without decline in quality. This system encourages uncontaminated, pure materials to be used to promote ease of recycling or decomposition. 
Goldsworthy (2014) discusses design for cyclability in relation to laser processing for textiles, proposing that synthetic textile products should be designed for recovery at end of life; that is, they should remain uncontaminated for repeated cycles of use and recycling. The design for cyclability proposal has relevance to the study presented in this paper, which aimed to develop laser techniques for creating three-dimensional textiles that negate additive processes. Emerging developments in textile recycling mean that high quality fibre-to-fibre recycling is becoming a commercial possibility for textile manufacture (GreenBlue 2017). Therefore, keeping fabrics pure to one fibre type opens the possibility for outcomes to be recovered and recycled responsibly, increasing the overall sustainability of the end products.

Emergent technological and engineering advancements are adding to sustainable improvements in manufacture. Literature in the field suggests new technologies are one way to boost the sustainability of production in the realm of fashion and textiles (Scaturro 2008). Studies using Life Cycle Assessment have confirmed that alternate specialist technologies for textile and garment processing can offer commercially viable, more sustainable forms of production as well as encouraging local manufacture (Allwood et al. 2006). It is worth noting that efficiency in manufacture can provide attractive economic benefits for industry as well as environmental benefits, reducing waste through improved use of resources.

In addition to the environmental benefits, digital technology and production systems may also offer other commercial benefits: such as allowing economic production of smaller batches, including madeto-order production and customisation at supplier or even consumer level, without the need for expensive setup costs (Allwood et al. 2006). This is widely referred to as agile manufacturing: a manufacturing paradigm that enables companies to meet market demands rapidly and responsively (Vinodh \& Kuttalingam 2011).

The laser is one such agile technology that has sustainable potential. It is a digital technology that offers dry processing and potential for combining processing stages, for example, laser cutting combined with laser processed surface design could reduce the number of separate steps necessary to produce garments. Combining the environmental, economical and practical processing advantages of laser technology with new techniques for textile design that adhere to circular material systems has potential to support sustainability in the field of fashion and textiles. This study focuses on a new, reversible textile surface design technique that will be discussed in relation to its benefits for circularity. 


\subsection{Interdisciplinary design research in contemporary textiles}

While textile design as a subject may require an interdisciplinary mind-set, it can often be difficult for designers to access the scientific and technical facets of textiles, production or technology without a technical background. Typically, areas of textile design and textile engineering operate separately within the textile industry. However, it has been recognised that collaboration and connections between fields can facilitate innovation. A report for the Crafts Council (KPMG 2016) notes the ability for craft to support cross sector innovation and discovery in the UK. This places textile designers in a significant position to balance craft, design and technology to facilitate material innovation. Literature and research that bring together practical, scientific and aesthetic strands in equal depth have been infrequent. However, an increasing movement of textile research for material innovation has seen a shift in the role of textile designer, from one of design practitioner to one that necessitates interdisciplinary expertise to communicate across diverse and collaborative scenarios. The shift is exemplified in contemporary design research, with many recent examples that notes the significance of bringing together creative and scientific approaches (Morgan 2017; Paine et al. 2017; Akiwowo et al. 2014; Earley \& Hornbuckle 2017; Ellams et al. 2014). The research presented in this paper summarises an in-depth study that brings together interdisciplinary features.

\subsection{Three-Dimensional Textiles}

In the design and construction of commercial and industrial textiles, three-dimensional surfaces are often used to provide beneficial properties to the fabric. Many traditional constructed, woven and stitched textile patterns exist for the enhanced properties they can offer for textile end-use: for example, increased absorption and insulation, strength and compression properties. Functional finishes for textiles that were originally designed for high performance have become synonymous with high quality style, leading to their adoption in fashion and trend led textile products for aesthetic appeal (Braddock and O'Mahony 1998; Salazar 2008).

Traditionally, three-dimensional effects can be added during the construction of textiles such as weaving, or in the finishing phases through additive embroidery and stitching techniques. Some wettechniques such as devoré, flocking, felting and shibori can also provide three-dimensional effects. Heat and heat-setting methods have long been used for creating three-dimensional forms on synthetic substrates. Textile practitioners who have investigated heat or laser effects to produce threedimensional textile outcomes include: Nigel Marshal's vacuum formed textiles (Braddock and O'Mahony 1998); Isabel Dodd's (1999) sculptural velvet accessories; Janette Matthews' (2009) laser 
assisted origami textiles; and Kate Goldsworthy's (2014) laser bonded synthetics. The potential to use a $\mathrm{CO}_{2}$ laser to heat-set pre-determined shapes in synthetic textiles had not been previously explored.

\subsection{Effect of heat on synthetic polymers}

To understand the changes taking place on polyester and nylon substrates due to laser irradiation, it is useful to review the role that heat plays in the formation and processing of synthetic textiles.

When a synthetic fibre is formed, crystalline and non-crystalline (amorphous) regions are present. The fibres undergo processing known as cold drawing, creating an ordered orientation, that increases crystallinity and strength as shown in Figure 1. If the fibre were to be heated above the glass transition temperature, molecular movement in the amorphous regions would result in the fibre returning to the undrawn state, reversing the cold drawing process and resulting in shrinkage (Horrocks \& Anand 2000). To prevent shrinkage of synthetic textiles during use, a heat setting process is used to fix fabric dimensions. The textile, with fibres in their drawn state, is held in place using stenter apparatus. When the textile is heated above glass transition temperature, molecular movement occurs as before, however shrinkage is not permitted due to the positioning on the tension frame. Instead, the relative orientation of molecules in the amorphous regions begin to crystallise in the stretched position (ibid). The dimensions of the fabric after heat setting will be stable up to the heat setting temperature. That is, the fabric will not now shrink when heated above the glass transition temperature. However, if the fabric is heated beyond the heat setting temperature shrinkage will occur as the fabric returns to its pre-set state. Could these thermal characteristics be used to engineer design features and functional material properties into textile materials that could later be recovered for re-use at a later phase in their life cycle?

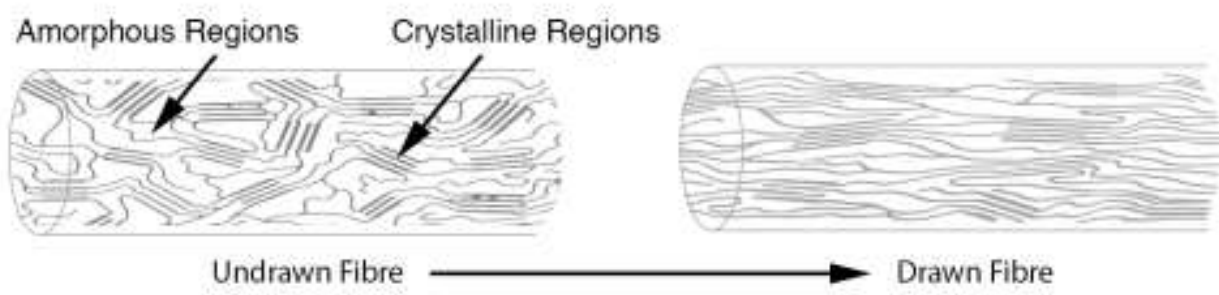

Figure 1. Effect of drawing on polymer fibres during the spinning process (C) Laura Morgan 2016

Montazer and Bahtiyari have reported reduction in the crystallinity of polymers after laser irradiation for both polyester (Montazer 2011) and polyamide fibres (Bahtiyari 2011). It can be concluded that the reported change in crystallinity is related to change in temperature. When the thermal energy of 
the laser heats the synthetic fibres above their glass transition temperature, or indeed above their heat-setting temperature, some shrinkage may occur. If the power is further increased and the temperature of polymers is heated towards melting point, molecules in the crystalline regions are weakened by thermal energy and the polymer begins to melt further increasing the amorphous regions in the laser irradiated areas. Thereby revealing the laser as a potential tool for precise, targeted application of heat that may be able to control the amorphous/crystalline properties of synthetic materials.

\section{Methods: Interdisciplinary textile design research process}

Interdisciplinary collaboration in this practice-based research took the form of material-led and digital textile design practice, quantitative scientific enquiry and qualitative industry feedback. In order to achieve this, the research was carried out across engineering, chemistry and art departments, drawing on specialist knowledge from optical engineering, dye and polymer chemistry, material science and design. Industrial stakeholders within contract interior textile manufacturing and performance swimwear provided feedback to ensure commercial viability. This crossing of disciplines led to a thorough investigation of the subject maintaining rigor across the interdisciplinary fields of study, found to be essential in developing the Laser Shibori technique. (Morgan 2017).

Figure 2 maps the inputs, practice and output of the interdisciplinary textile design research process used in this study that lead to material innovation. The approach used to develop and examine new laser techniques made use of the first author's experiential knowledge of textiles and design, whilst also drawing on material science. This material-led approach in the initial stages of the research provided proof of concept that was developed and tested with creative design practice together with technical and scientific procedures to measure and explain the process. Quantitative data and mathematical interpretations were used to predict and control the effects, without which, the results achieved would remain happy accidents or one-off effects that could not be replicated, limiting the potential for innovation. Instead, command of the necessary parameters for predicting the threedimensional effects enabled design flexibility through controlled design outcomes suitable for technology transfer across industrial laser systems. Design practice was used to develop and test the aesthetic and tactile qualities that could be achieved. Examining varied laser moulded shapes and their resulting forms on textile substrates identified a library of decorative and functional design and application opportunities to support sustainable material innovation. 


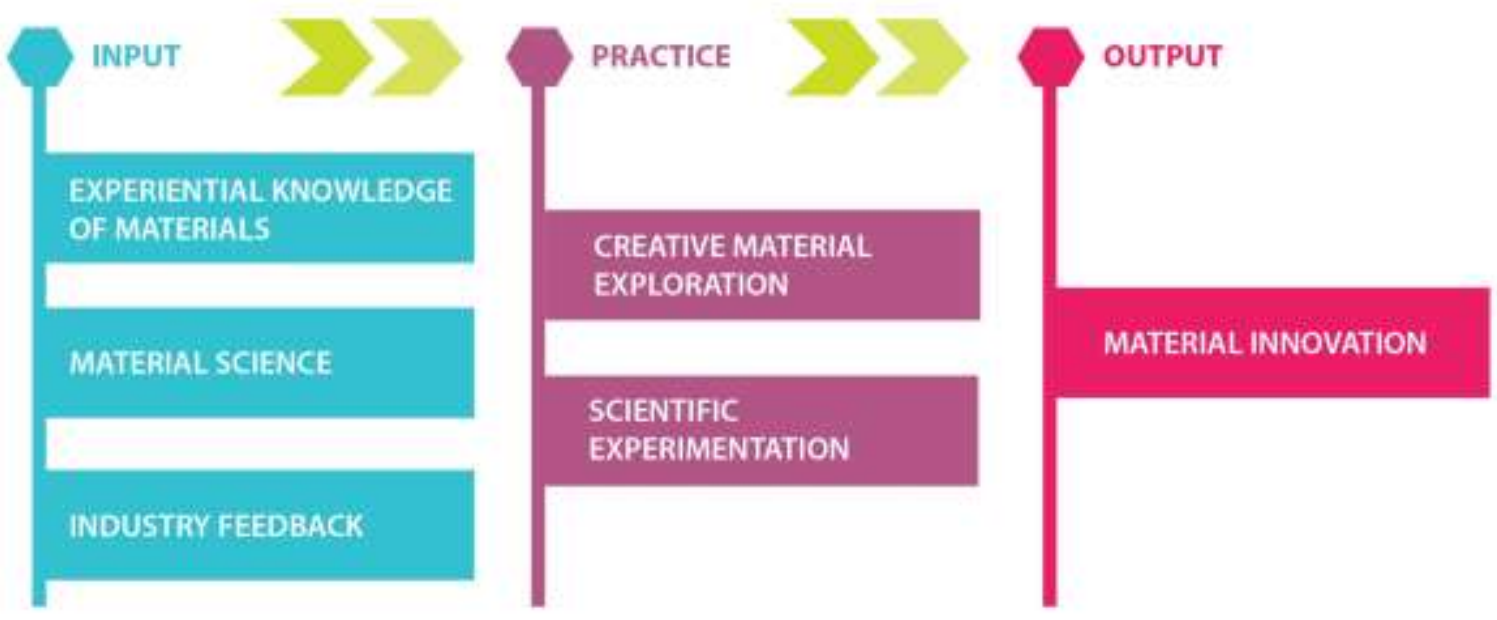

Figure 2. Interdisciplinary textile design research process for material innovation (c) Laura Morgan 2018

\subsection{The development of Laser Shibori}

Using the interdisciplinary textile design research process for material innovation described in Figure 2, Figure 3 shows the specific processes used in the development of the Laser Shibori technique. The author's experiential knowledge in weaving processes provided a familiarity with achievable design effects facilitated by altering tension in the production of constructed textiles. When this expertise was combined with material science knowledge gained from a literature review on the effect of heat in forming and finishing synthetic fibres, it was hypothesised that three-dimensional surface design effects may be achieved by laser irradiation of a fabric substrate under strain.

The creative approach used textile design practice to explore design effects and application opportunities of the moulding technique, while a scientific approach provided a measurable means to calculate, predict and control the laser moulding effects for designing the three-dimensional surfaces of Laser Shibori. Important parameters were identified and explored through experimentation, leading to a system for predicting the three-dimensional effects to enable controlled design outcomes. Altering parameters offered a range of three-dimensional textile design features and opportunities as listed in Figure 3. 


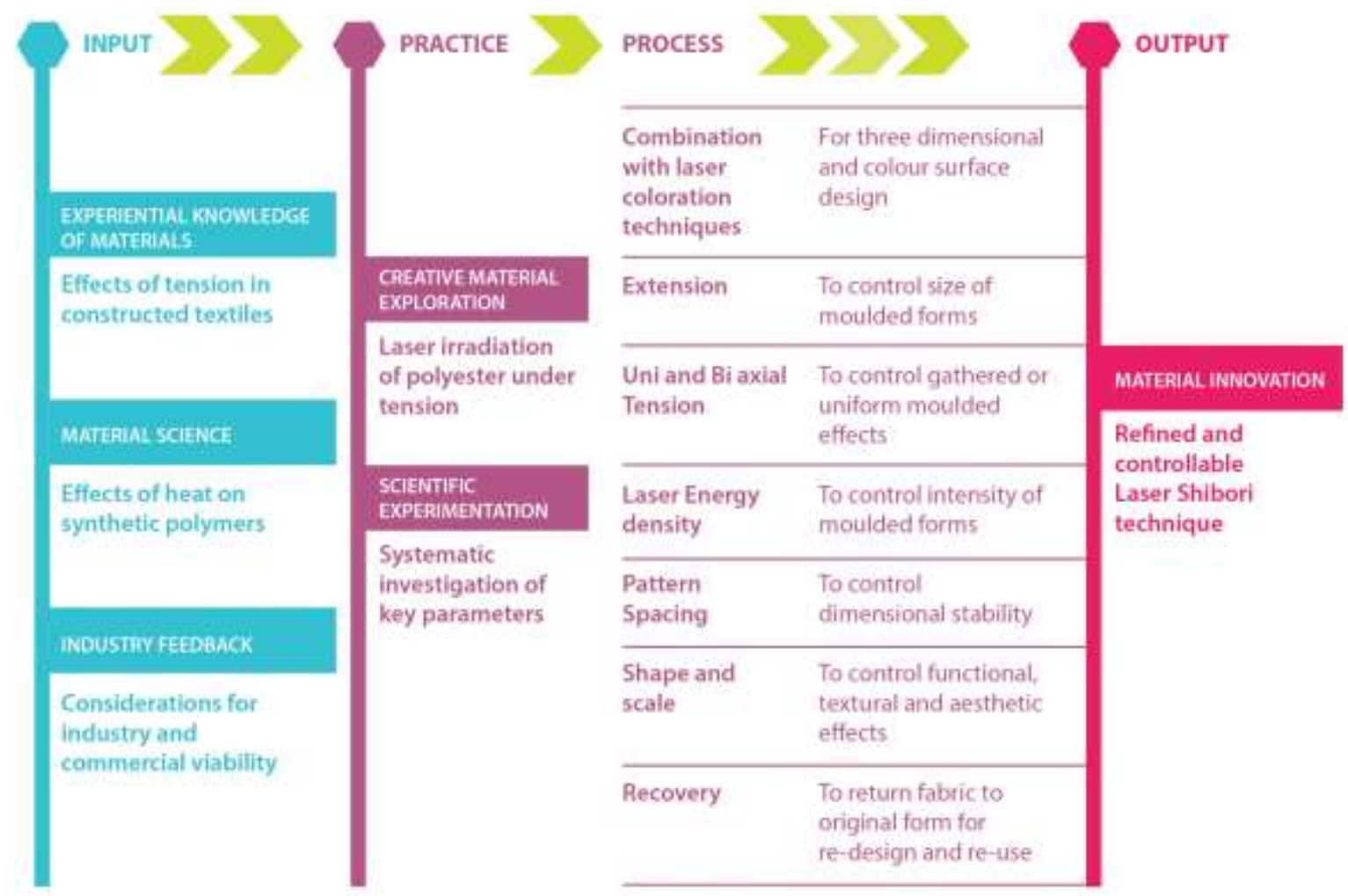

Figure 3. Interdisciplinary textile design research process leading to a refined Laser Shibori Technique (C) Laura Morgan 2018

The laser moulding technique was developed using the photothermal energy from an infrared laser to induce a heat setting effect on polyester substrates. A knitted $100 \%$ polyester fabric was used, provided by an industrial partner to the project. The fabrics were held under tension using lab-scale stenter apparatus, which allowed tension force and extension to be recorded. The laser used in this study was a Synrad Carbon Dioxide $\left(\mathrm{CO}_{2}\right)$ source laser operating in the far infrared spectrum at a wavelength of $10.6 \mu \mathrm{m}$. Digital control of the laser beam through computer aided design (CAD) software allowed laser irradiation of targeted areas on the test material. On release from tension, the laser irradiated areas created three-dimensional protrusions from the surface of the cloth (figure 4). A laser dye fixation method, also developed by the author and referred to as peri-dyeing, considered the laser as an on-the-spot dye fixation tool that can be used for precise coloration and patterning of textiles (Morgan 2016). When combined with the laser dyeing technique, laser moulding created a three-dimensional relief colour pattern. The laser energy density delivered to the marked areas provided a sufficient thermal reaction for dye diffusion and heat setting to take place simultaneously. The design opportunities provided by combining the two processes increase design flexibility. For example, multi-coloured imagery and pattern could be enhanced by three-dimensional elements in targeted areas of the design. The results could be compared to shibori dyeing; a resist dyeing process where dyeing and three-dimensional effects are combined. The Laser Shibori provided its own unique 
aesthetic effect, offering control and digital image making, with a level of precision and repeatability that cannot be achieved with existing shibori processes or alternative textile production techniques (figure 5).

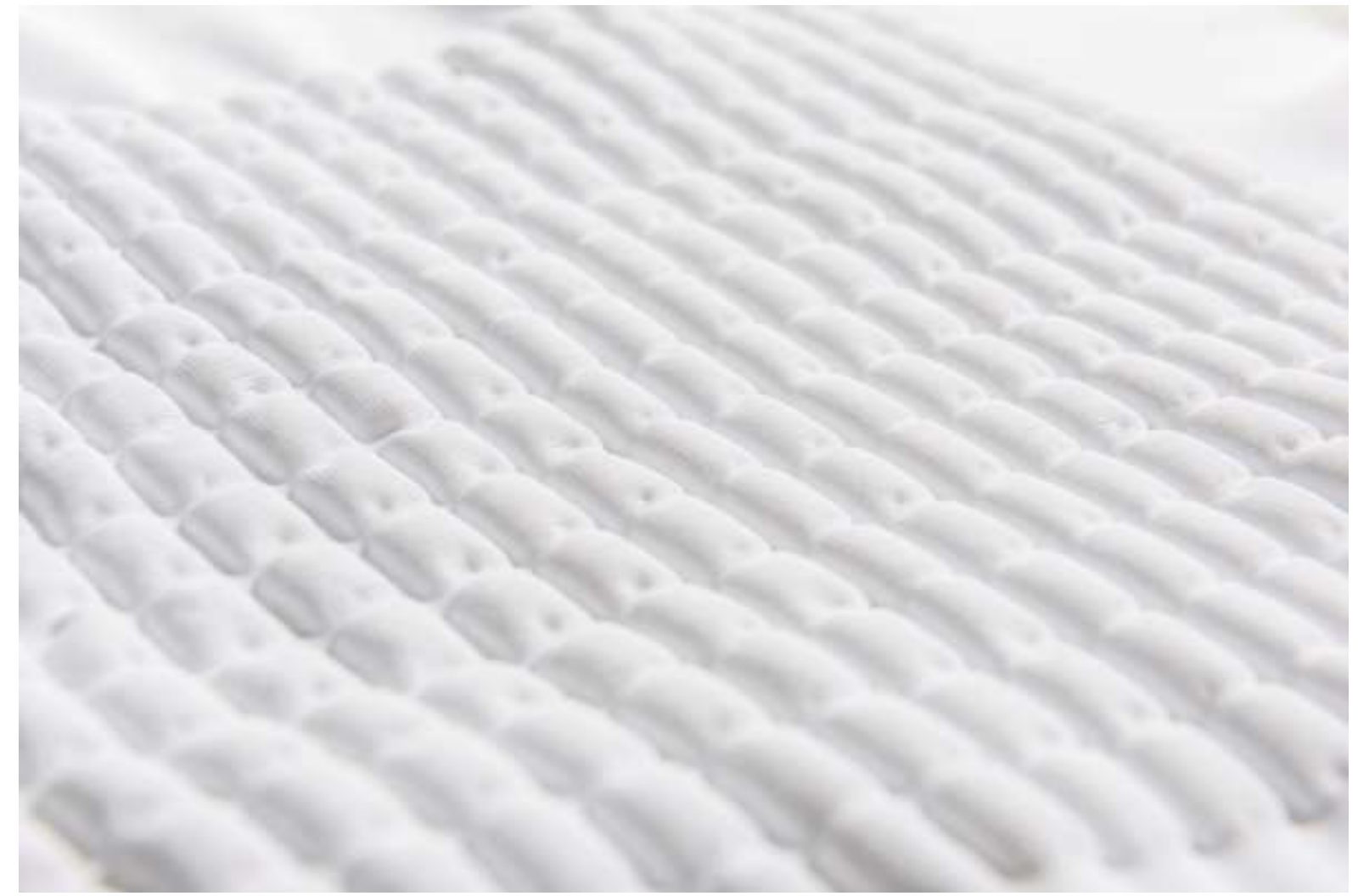

Figure 4. Laser moulded polyester sample @ Laura Morgan 2016

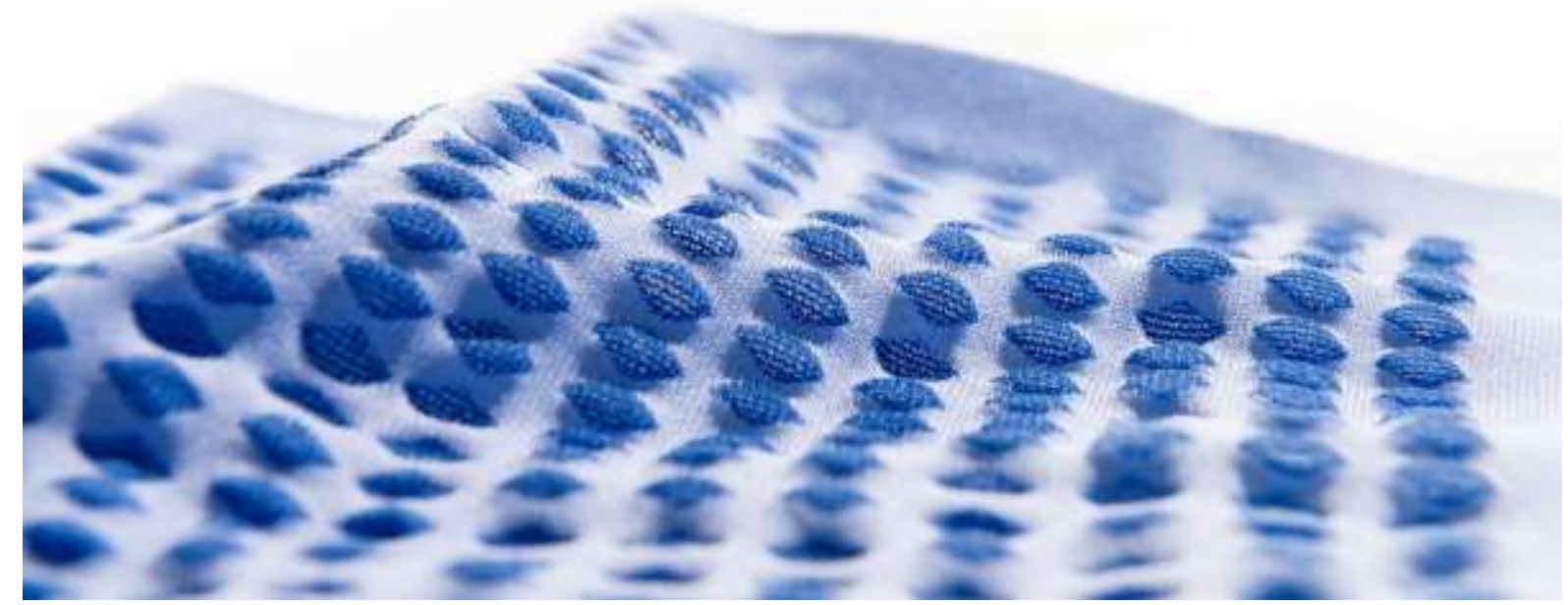

Figure 5. Laser Shibori: Laser moulded colour sample (c) Laura Morgan 2016 


\section{Controlling three-dimensional laser moulded features}

This section provides a summary of the technical controls and the achievable effects from utilising different parameters to manipulate scale and shape of three-dimensional textile surfaces. In addition, it will discuss the potential to offer new or enhanced fabric properties, design features and application opportunities.

The laser moulding phenomenon can be explained with reference to the known effect of heat on polymer states and confirmed through microscopic surface observation. When the textile was held under tension in a stretched position, and irradiated by the laser to temperatures above glass transition temperature, the degree of crystallinity increased under fixed tension. This acted to heatset the laser-irradiated area in the extended dimensions. When released from tension, the untreated cloth returned to its original dimension while the laser irradiated areas did not. This provided an excess of fabric in the laser irradiated areas, which is forced upwards leading to a protrusion on the fabric surface.

\subsection{Effect of tension on three-dimensional moulding}

An experiment was conducted to determine if tension could be used as a means to control threedimensional design effects and secondly to discover the effect of altering tension on the intensity of three-dimensional forms. An area of polyester fabric was laser irradiated using pre-determined laser parameters. The process was repeated applying tension by increasing force in equal increments and recording the extended length of the working area. On release from tension, the textile sample contracted and the new dimensions of the laser irradiated area were recorded. A graph of the recorded figures was plotted resulting in a straight line (Figure 6). This suggests a directly proportional relationship between the tension applied to the base fabric and the resulting size of the laser moulded shapes after release from tension.

The graph can be read to determine the percentage by which the laser-irradiated area contracts when processed at a given extension. The diagram in Figure 7 shows an example: a sample extended by $50 \%$ of its original dimension and laser-irradiated across $(5 \mathrm{~cm})$ will contract by $29 \%$, resulting in a relaxed length of $3.6 \mathrm{~cm}$. The graph was used to predict and control the size of moulded features based on design requirements in later sampling. The diagram displays how the fabric retains the laser-irradiated dimension on the surface, while the working area of the sample contracts, resulting in a threedimensional protrusion from the surface of the cloth. After being released from tension, the 
dimension of the entire working area returned to equilibrium in its original relaxed position, confirming that laser treatment did not affect the bulk elastic properties of the sample, only that of the laser-treated area.

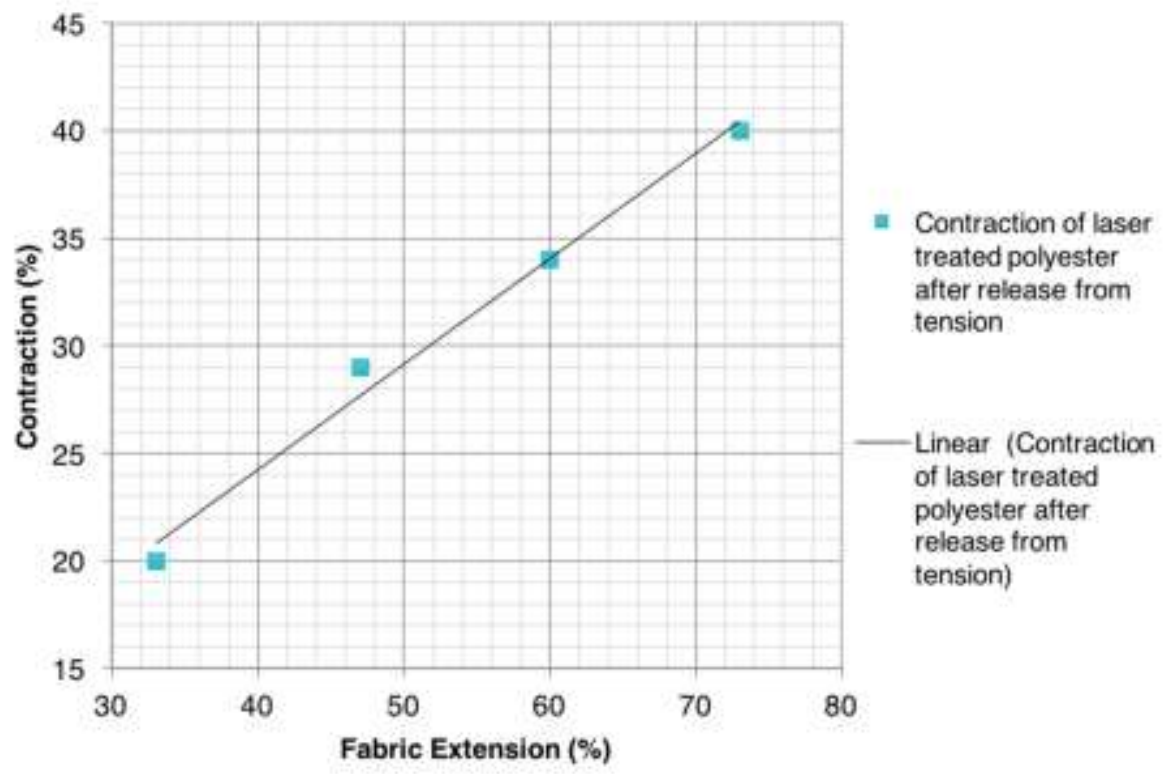

Figure 6. Graph showing fabric extension under tension against contraction after release from tension of laser irradiated polyester fabric (c) Laura Morgan 2016

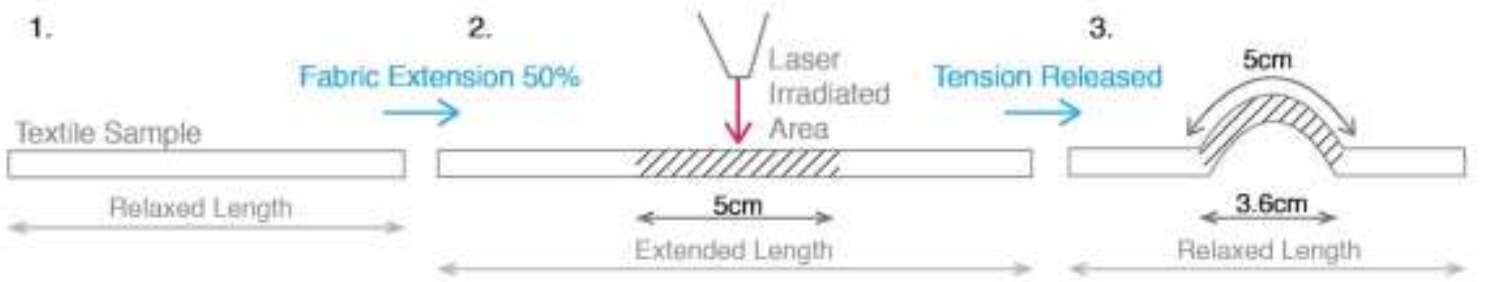

Figure 7. Diagram showing the effect of tension on laser moulding (C Laura Morgan 2016

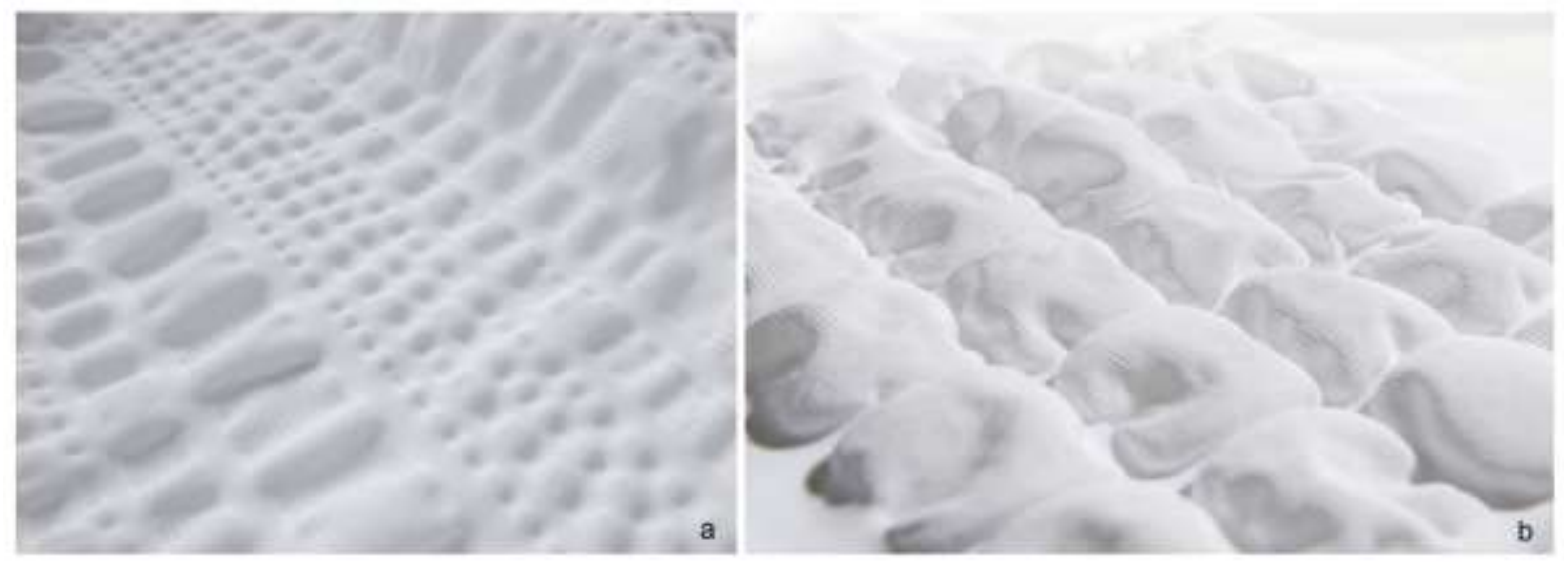

Figure 8. a) Seer sucker effects achieved by tension applied to one axis. b) Full shapes achieved by even bi-axial tension (C) Laura Morgan 2016 
The application of uni axial or bi axial tension (force applied to one or two directions) was used to control the vertical and horizontal effects achieved on the fabrics. For example, gathered effects akin to seer sucker were achieved by tension applied to one axis. While fully formed shapes were achieved by even bi-axial tension as shown in Figure 8 .

\subsection{Altering laser energy density}

Laser energy density is a measurement used to quantify the energy delivered to a substrate via the laser beam. Also referred to as laser fluence, the measurement takes into consideration multiple parameters that can affect laser processing to provide a single number. This allows replication of results across varied laser systems, where power outputs and velocity capabilities may differ from one machine to another.

In order to establish optimal laser settings and to examine the effect of altering energy density, a range of parameters were laser marked on polyester fabric held under tension at a fixed extension. Optimal parameters for the purposes of this study can be defined as those that provide threedimensional design effects, whilst causing minimal thermal damage to the fabric to preserve mechanical properties. While the results showed no change in the size of the affected area after laser irradiation, the intensity of the three-dimensional effect increased with increasing laser energy density, up to the point of ablation. At higher laser energy densities increased heat-setting temperatures resulted in a more solid, crystalline structure. Therefore, the laser irradiated area held its processed shape more firmly. At lesser laser energy densities, the effect was less solid, only partially holding the laser processed shape. It was found that altering parameters could be used to achieve a scale of three-dimensional qualities from a slight undulation in the fabric surface at lower energy densities, to solid protruding shapes at higher energy density outputs. This is explained in the diagram in Figure 9.

Within the identified range of energy densities, the use of lower energy densities for laser moulding have potential to create subtle relief effects while higher powers were used to create dramatic threedimensional surface qualities. 


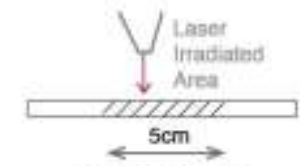

Extended Length

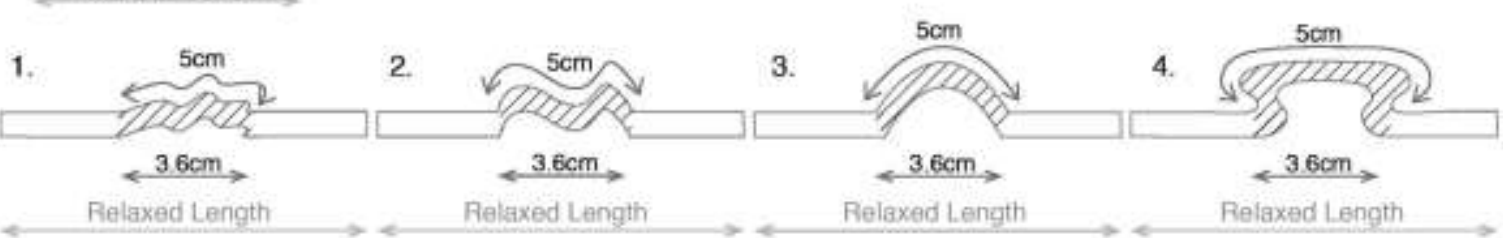

Increasing Laser Energy Density from 1 - 4

Relaxad Lanoth

Figure 9. The effect of laser energy density on laser moulding (c) Laura Morgan 2016

\subsection{The effect of pattern spacing on laser moulding}

During sampling, it was noted that some patterns affected the dimensional stability of the entire working area, resulting in an undesirable distorted surface in addition to the intentional 3D effects. It was observed that the space separating laser irradiated areas was as important in the creation of a successful moulded sample. The non-irradiated areas of the textile must retain an adequate structure akin to a warp and weft for the structural integrity of the textile sample to remain intact. This is shown diagrammatically in Figure 10. A laser irradiated pattern without clear vertical and horizontal spacing affects the textile sample to the same extent as laser processing the entire working area resulting in distortion. However, a pattern that allows clear vertical and horizontal lines of space to traverse the design, results in a sample that remains intact and flat, with only the laser-treated shapes providing 3D protrusions from the cloth.
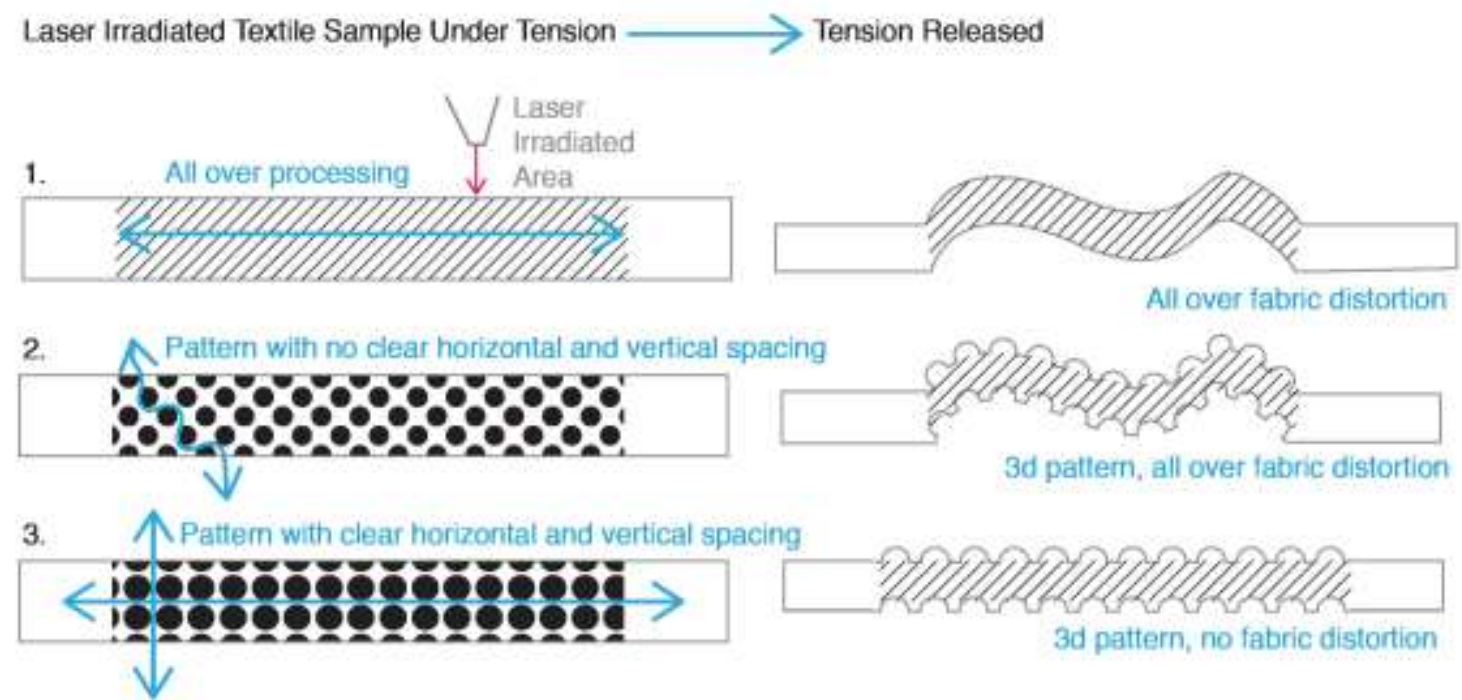

Figure 10. The effect of pattern spacing on laser moulding (C Laura Morgan 2016 


\subsection{Effect of scale and shape on laser moulding}

The effect achieved by altering scale and shape of three-dimensional textile surfaces was investigated. To do this, traditional constructed textile patterns including stripes, hopsack, basket weave, honeycomb and geometric shapes were used to examine the design and application potential, and the aesthetic and functional effects that could be achieved by laser moulding. The use of forms inspired by traditional textile textures presented a rich library of aesthetic and tactile patterning opportunities borrowed from traditional textiles structures as well as the potential performance benefits they may offer, including thermal insulation, airflow, breathability and tailoring functionality. Parameters established in the previous sections were used to facilitate the desired three-dimensional surfaces including, weave and stitch-inspired effects; shaping effects and texturing effects.

Laser-moulded weave and stitch inspired effects resulted in a variety of laser-moulded textile design samples. The samples in Figure 11 showed potential to offer aesthetic affect and increased functionality in textile products including: Geometric hopsack and basket weave patterns providing optical illusion effects by emulating traditional woven textures (b); Laser moulded honeycomb, a structure traditionally used to provide increased absorption and insulation properties in textiles (c); Large scale laser moulding to create dramatic three-dimensional moulds and surface qualities akin to quilting (a); and seersucker, often used in clothing to offer heat dissipation in hot weather garments, where rippled areas of the textile remain loose to facilitate air circulation during wear (Figure 8a).

Laser-moulded shaping effects have potential to offer benefits for finished textile product applications such as ease of movement at joints or engineered fitting opportunities in the design and tailoring of garments. For example, gathered, ruched or pleated finishes (Figure 11g-h). These effects provide visual interest to a textile product or add functionality such as freedom of movement to the wearer. The laser-moulded material in Figure 11 (e-f) exhibits the properties of a soft spring which could have possible relevance for clothing, architectural or medical textile applications. Large scale threedimensional forms (d) show potential for shaped garments such as laser moulded cups onto a textile for intimate apparel or sportswear. Targeted placement of laser moulding to enable engineered fitting or shaping for moulded garment pattern pieces could offer an exciting opportunity to develop custom fitting with the goal of starting from a blank standard fabric and adding targeted properties or shaping based on requirement. 


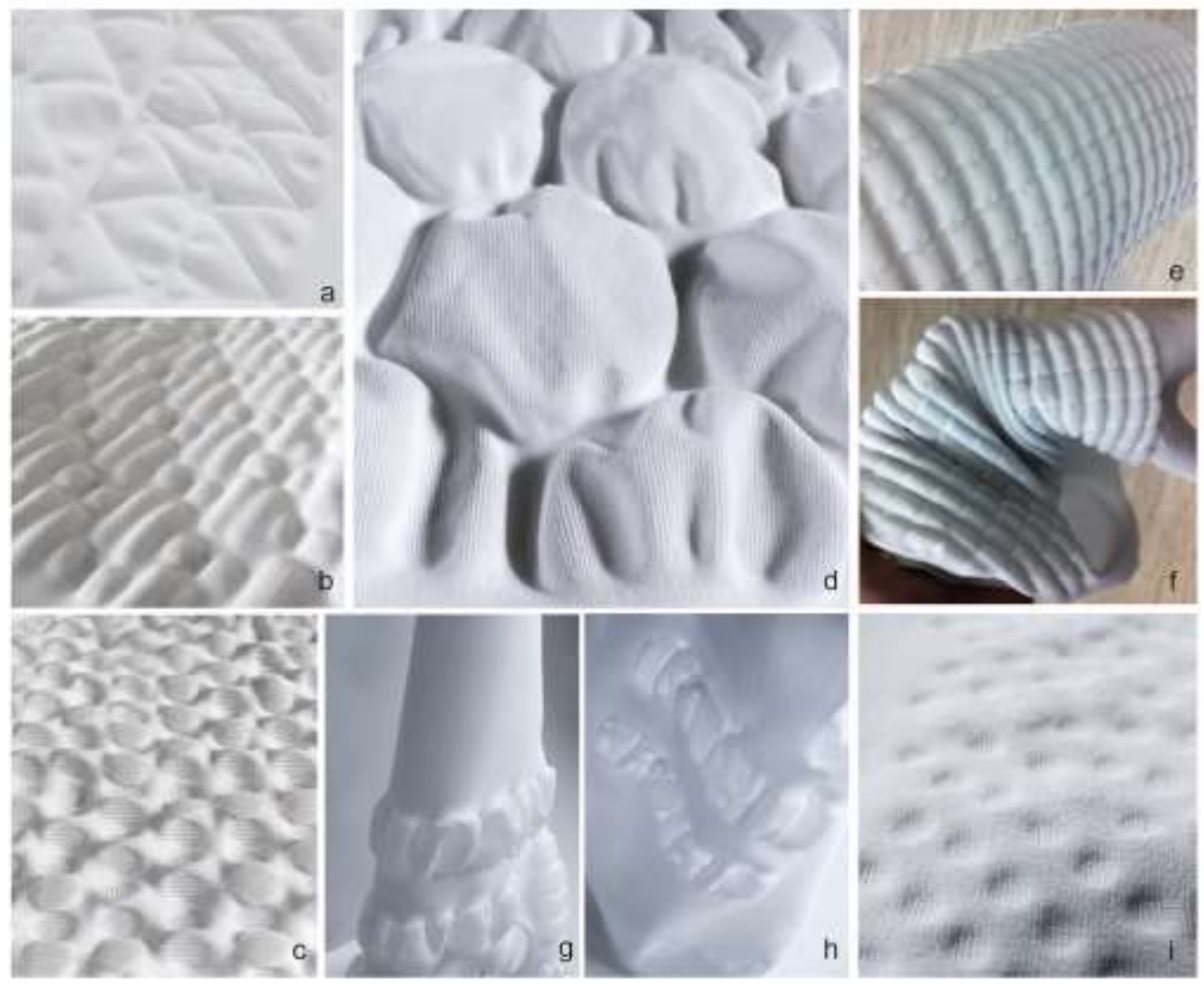

Figure 11. Laser moulded design samples inspired by traditional textile constructions: a) quilted effects; $b$ ) basket weave; c) honeycomb; d) large moulded forms; e- f) pleated effects; g-h) gathering effects; i) small scale dimpled effects (C) Laura Morgan 2016

Laser-moulded texturing effects add visual interest and changes in tactile qualities. In Figure 11 (i), an example of small-scale patterning shows a dimpled fabric texture. Patterns of this nature have been used within performance sportswear to provide aerodynamic texturing (Nike 2012). With a beam size of $0.03 \mathrm{~cm}$, laser moulding could occur on a microscopic scale. Micro-patterning or micro-texturing has the potential to alter tactile properties: a subject for further investigation.

\subsection{Recovery}

Heat moulding is known to remain effective up to the temperature at which the heat setting took place. Beyond that temperature fixed dimensions of the fabric are likely to become unstable, resulting in a loss of the heat-set properties (Horrocks \& Anand 2000).

On washing the Laser Shibori textiles above temperatures of $100^{\circ} \mathrm{C}$, it was found that the samples did not retain their three-dimensional forms, instead returning to their original flat surface. This would allow relative ease of material recovery, while ensuring the materials retained their moulded forms at conventional consumer washing temperatures. Recovering the textile to its original state offers 
significant potential for goods to be designed and re-designed, prolonging their primary use before returning to the industrial loop to be recycled.

\section{Advantages for Sustainability, Manufacture and Recovery}

In this study, the laser moulding technique was shown as an effective method for creating threedimensional forms on pure polyester or nylon knitted textiles. The technique could be used for surface design and to engineer functional properties such as comfort, breathability, insulation and aerodynamic texturing to textile goods. Combining three-dimensional laser moulding with dyeing processes resulted in an effect similar to shibori dyeing. However, the Laser Shibori provided unique design aesthetics, offering control, with a level of precision and repeatability that cannot be achieved with existing shibori processes or textile production techniques.

The use of laser technology to create three-dimensional, dyed textile forms presents efficient processing advantages over traditional methods. Unlike regular textile embossing equipment, the laser does not require moulds or plates to be cast for each new design. Unlike the relief effects created through weaving, the laser does not require complicated loom set up to produce three-dimensional forms. Coloration for Laser Shibori was attained by combining laser moulding with a laser peri-dyeing method that displays potential savings in energy, water and resource consumption compared to immersion dyeing procedures (Morgan, 2016). The Laser Shibori effects offer ease of pattern change through digital generation of designs. This in turn would allow targeted, direct-to-garment processing opportunities on textile blanks or engineered garment pattern pieces, adding additional properties to a textile, post-construction.

The opportunity for digital laser processes to move the design stage further down the production cycle could allow for late stage decisions and design flexibility, providing a responsive approach to design and manufacture. For manufacture and supply chains, responsive or agile manufacturing offers reduced lead times and minimum orders to reduce surplus stock and to minimise or eliminate the creation of excess waste of textile goods; in addition, it may facilitate bespoke or customised production opportunities.

The design of systems to compliment and facilitate circular material processing that have additional efficiency benefits for manufacture may encourage uptake of more sustainable technologies and processing techniques in the textile industry. Laser moulding is a dry and efficient process that can 
make use of existing $\mathrm{CO}_{2}$ laser processing equipment and does not require additional materials, such as thread for stitching. Therefore, using the technique for surface design effects could eliminate the need for additional embellishment for decorative and functional textiles. The laser features remain fixed at conventional laundering temperatures. However, when the textile is heated beyond the temperature with which it was set by the laser, the effects are reversed and the cloth returns to its original condition. The reversible features show potential ease of recovery, redesign and re-use. While the use of synthetic mono materials may allow textile products to be recycled effectively in a closed loop lifecycle to facilitate circularity as shown in Figure 12. Further work to examine the properties of the treated material after recovery would identify constraints and scope in relation to repeated cycles of use and re-design.

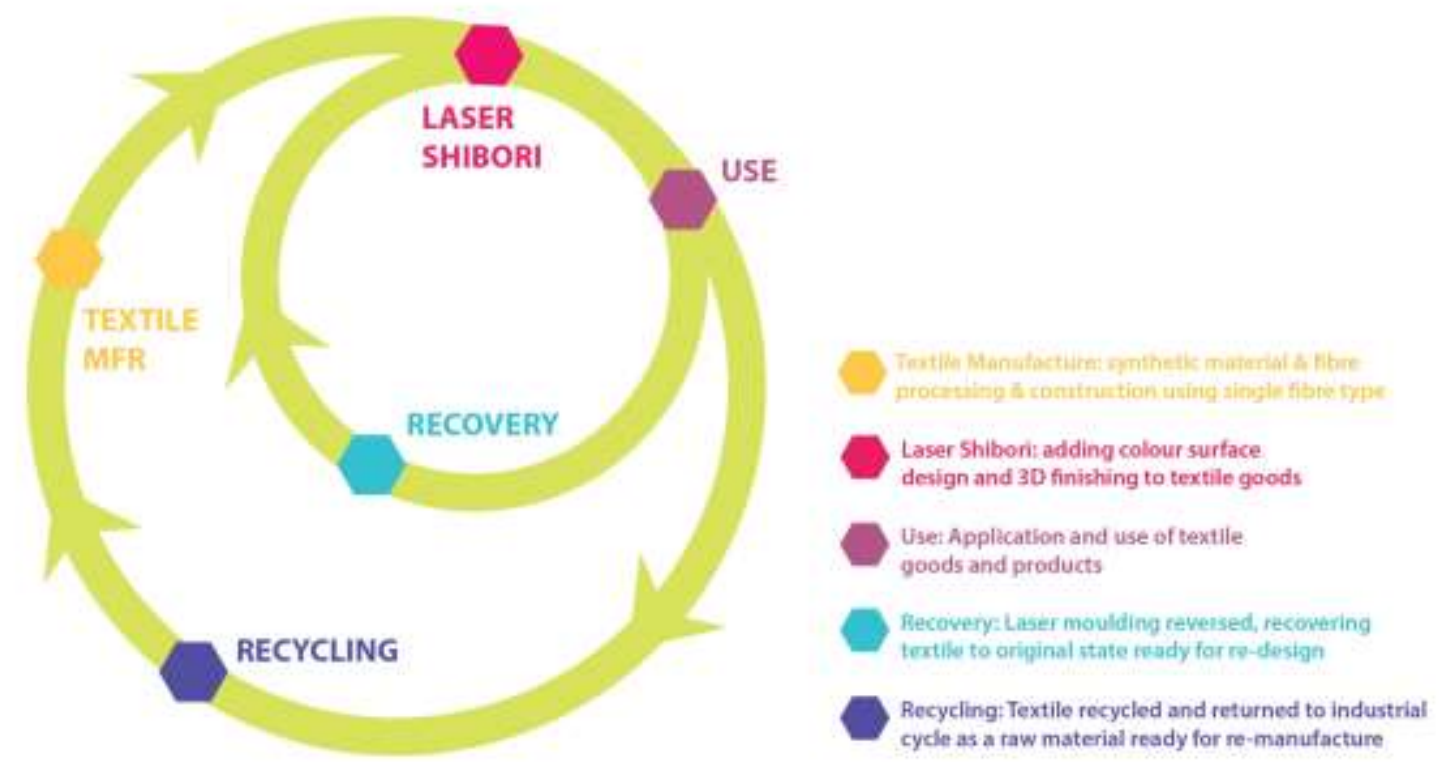

Figure 12. Laser Shibori in a circular textile lifecycle (C) Laura Morgan 2018

\section{Conclusion}

This paper summarised a new method for moulding synthetic stretch textiles. A selection of laser moulded samples was presented, discussing the functional and aesthetic possibilities for textile design and avenues for further investigation. The technique, which used the photothermal properties of the $\mathrm{CO}_{2}$ laser, allowed accurate three-dimensional moulding of synthetic fabrics without the use of pattern moulds.

The laser moulding technique was used to design accurate surface architectures providing threedimensional design features for textile materials. Combining the technique with dyeing processes 
resulted in an effect akin to shibori. Unlike the traditional craft practice, Laser Shibori offered precise control, repeatability and a unique aesthetic. Designs were created digitally and could be changed effortlessly. The method allowed decoration to emerge from the structure of the cloth without contaminating the mono-material fibres. This in turn may allow the cloth to be recovered or recycled easily, adhering to a closed-loop system for a sustainable textile lifecycle.

The study demonstrated the benefit of interdisciplinary study, synthesising design and science to support sustainable material innovation. The synthesis of the scientific and creative approaches proved essential in creating the laser technique as a design tool with potential to support a circular textile economy in three dimensions: through efficiency, agility and recovery in textile manufacture, finishing and re-design.

\section{Acknowledgements}

The authors would like to thank the Arts and Humanities Research Council (UK) for funding the collaborative LEBIOTEX Project (AH/J002666/1) between De Montfort University and Loughborough University, in addition to the support from the industrial partners Camira, Speedo and Teresa Green Design. 


\section{References}

Akiwowo, K, Kane, F, Tyrer, J, Weaver, G and Filarowski, A. 2014. "Digital Laser-dyeing for Polyester Fabrics." Journal of Textile Design Research and Practice, 2(2), pp.133-152.

Allwood, J., Laursen, S., Malvido De Rodríguez, C. and Bocken, N. 2006. Well Dressed? The Present and Future Sustainability of Clothing and Textiles in the United Kingdom. Cambridge: University of Cambridge.

Bahtiyari, M.I. 2011. "Laser Modification of Polyamide Fabrics." Optics and Laser Technology, 43(1) pp. 114-118.

GreenBlue. 2017. Chemical Recycling: Making Fiber-to-Fiber Recycling a Reality for Polyester Textiles. Web: 18 February 2018.

http://www.sustainablebrands.com/digital_learning/tool/chemistry_materials_packaging/making_fi ber--fiber_recycling_reality_polyester_.

Braddock, S and O'Mahony, M. 1998. Techno Textiles: Revolutionary Fabrics for Fashion and Design. London: Thames \& Hudson.

Dodd, I. (1999) "Velvet Scarf with Hand Printed Large Spot." Crafts Council. Web: 30 November 2015. http://collections.craftscouncil.org.uk/object-t151

Earley, R. and Hornbuckle, R. 2017. "Postcards from The Edge: Trash-2-Cash Communication Tools used to Support Interdisciplinary Work Towards a Design Driven Material Innovation (DDMI) Methodology." IOP Conference Series: Materials Science and Engineering, 254.

Ellams, D. L., Christie, R. M. and Robertson, S. 2014. "An Approach to Sustainable Coloration of Lyocell Fabrics by Screen Printing Using Extracts of Leaves and Bark from Eucalyptus." Coloration Technology, 130: 48-53.

Forum for The Future. 2007. Fashioning Sustainability; A Review of the Sustainability Impacts of the Clothing Industry. London: Forum for The Future.

Goldsworthy, K. 2014. "Design for Cyclability: Proactive Approaches for Maximising Material Recovery." Making Futures Journal, 3 (2014) 249.

Horrocks, A. and Anand, S. 2000. Handbook of Technical Textiles. Cambridge: Woodhead.

KPMG. 2016. Innovation Through Craft: Opportunities for Growth: A Report for the Crafts Council. London: KPMG.

Matthews, J., Kavanagh, T.J. and Tyrer, J.R. 2009. "Laser Assisted Design for Three-Dimensional Pleated Structures." In ICALEO 2009 - 28th International Congress on Applications of Lasers and Electro-Optics, Congress Proceedings, pp.400-405.

McDonough, W. and Braungart, M. 2002. Cradle to Cradle: Remaking the Way We Make Things. New York: North Point Press.

Montazer, M., Javad, S. and Harifi, T. 2011. "Effect of $\mathrm{CO}_{2}$ Irradiation on Various Properties of Polyester Fabric: Focus on Dyeing." Journal of Applied Polymer Science, 124 (1) pp. 342-348. 
Morgan, L. 2016. Laser Textile Design: The Development of Laser Dyeing and Laser Moulding Processes for Sustainable Design and Manufacture. Doctoral Thesis: Loughborough University.

Morgan, L. 2017. "Interdisciplinary Textile Design Research for Material Innovation: Synthesising design, Science and Industry Collaboration." In Proceedings of Intersections: Collaborations in Textile Design Research Conference, 13 September 2017, Loughborough University London, U.K.

Nike. 2012. "Nike Unveils Track and Field Footwear and Apparel Innovations." Nike. Web: 10 January 2016. <http://news.nike.com/news/track-field-nike-pro-turbospeed-uniforms-and-nike-zoomspikes>.

Paine, H., Goldsworthy, K. and Baurley, S. 2017. "Evolutionary approach of a textile designer through cross-disciplinary research practice: A case study in the field of advanced methods for joining textiles." In Proceedings of Intersections: Collaborations in Textile Design Research Conference, 13 September 2017, Loughborough University London, U.K.

Scaturro, S. 2008. “Eco-Tech Fashion.” Fashion Theory, 112-4 (2008) 469-488.

Salazar, L. (ed) 2008. Fashion v Sport. London: V\&A Publishing.

The Ellen MacArthur Foundation. (2017) A New Textiles Economy: Redesigning Fashion's Future. <http://www.ellenmacarthurfoundation.org/publications>.

Vinodh, S. and Kuttalingam, D. 2011. "Computer-Aided Design and Engineering as Enablers of Agile Manufacturing." Journal of Manufacturing Technology Management, 22 (3) 405-418. 


\section{Figure Captions}

Figure 1. Effect of drawing on polymer fibres during the spinning process (C) Laura Morgan 2016 Figure 2. Interdisciplinary textile design research process for material innovation (־ Laura Morgan 2018

Figure 3. Interdisciplinary textile design research process leading to a refined Laser Shibori Technique (C) Laura Morgan 2018

Figure 4. Laser moulded polyester sample @ Laura Morgan 2016

Figure 5. Laser Shibori: Laser moulded colour sample (C) Laura Morgan 2016

Figure 6. Graph showing fabric extension under tension against contraction after release from tension of laser irradiated polyester fabric (c) Laura Morgan 2016

Figure 7. Diagram showing the effect of tension on laser moulding (c) Laura Morgan 2016

Figure 8. a) Seer sucker effects achieved by tension applied to one axis. b) Full shapes achieved by even bi-axial tension (C) Laura Morgan 2016

Figure 9. The effect of laser energy density on laser moulding (c) Laura Morgan 2016

Figure 10. The effect of pattern spacing on laser moulding (C Laura Morgan 2016

Figure 11. Laser moulded design samples inspired by traditional textile constructions: a) quilted effects; b) basket weave; c) honeycomb; d) large moulded forms; e- f) pleated effects; g-h) gathering effects; i) small scale dimpled effects (C) Laura Morgan 2016

Figure 12. Laser Shibori in a circular textile lifecycle @ Laura Morgan 2018 